\title{
Relative importance of an arbuscular mycorrhizal fungus (Rhizophagus intraradices) and root hairs in plant drought tolerance
}

\author{
Tao Li • Ge Lin • Xin Zhang • Yongliang Chen • \\ Shubin Zhang $\cdot$ Baodong Chen
}

Received: 24 January 2014 / Accepted: 7 April 2014 /Published online: 18 April 2014

(C) Springer-Verlag Berlin Heidelberg 2014

\begin{abstract}
Both arbuscular mycorrhizal (AM) fungi and root hairs play important roles in plant uptake of water and mineral nutrients. To reveal the relative importance of mycorrhiza and root hairs in plant water relations, a bald root barley $(b r b)$ mutant and its wild type $(w t)$ were grown with or without inoculation of the AM fungus Rhizophagus intraradices under well-watered or drought conditions, and plant physiological traits relevant to drought stress resistance were recorded. The experimental results indicated that the AM fungus could almost compensate for the absence of root hairs under drought-stressed conditions. Moreover, phosphorus (P) concentration, leaf water potential, photosynthetic rate, transpiration rate, stomatal conductance, and water use efficiency were significantly increased by $R$. intraradices but not by root hairs, except for shoot $P$ concentration and photosynthetic rate under the drought condition. Root hairs even significantly decreased root $\mathrm{P}$ concentration under drought stresses. These results confirm that AM fungi can enhance plant drought tolerance by improvement of $\mathrm{P}$ uptake and plant water relations, which subsequently promote plant photosynthetic performance and growth, while root hairs presumably contribute to the improvement of plant growth and photosynthetic capacity through an increase in shoot $\mathrm{P}$ concentration.
\end{abstract}

Tao Li and Ge Lin contributed equally to this work.

T. Li $\cdot$ X. Zhang $\cdot$ Y. Chen $\cdot$ B. Chen $(\triangle)$

State Key Laboratory of Urban and Regional Ecology, Research

Center for Eco-Environmental Sciences, Chinese Academy of

Sciences, Beijing 100085, China

e-mail: bdchen@rcees.ac.cn

G. Lin

Guiyang College of Traditional Chinese Medicine, Guiyang 550002,

China

S. Zhang

Institute of Plant Nutrition and Resources, Beijing Academy of

Agriculture and Forestry Sciences, Beijing 100097, China
Keywords Arbuscular mycorrhiza - Drought tolerance · Rhizophagus intraradices · Barley . Root hairs

\section{Introduction}

Drought as a major environmental stress imposes adverse effects on plant growth and development (Denby and Gehring 2005). Nevertheless, plants have developed several mechanisms at morphological, anatomical, and cellular levels to cope with drought stress (Bray 1997), such as the increased production of lateral roots (Jupp and Newman 1987) or the stimulation of root hair density and proliferation which can increase root surface and improve plant water uptake in arid areas (White and Kirkegaard 2010). Root hairs are singlewalled cells (Segal et al. 2008) where many water channels (Brechenmacher et al. 2009) and phosphate (Schünmann et al. 2004), nitrogen (Loqué et al. 2006), potassium (Lan et al. 2010), calcium (Lan et al. 2010), and sulfate transporters (Maruyama-Nakashita et al. 2004) are localized, all of which are beneficial to water and nutrient uptake by plants (Libault et al. 2010). It has been reported that root hairs enhance barley tolerance to phosphorus $(\mathrm{P})$ deficiency under water stress conditions (Brown et al. 2012). In addition, morphology of root hairs and release of compounds into the rhizosphere mediate the interaction of plants with beneficial soil microorganisms, such as arbuscular mycorrhizal (AM) fungi, which may help plants take up more water and nutrients under drought stress (Libault et al. 2010; Oldroyd and Downie 2006).

The AM symbiosis established with the majority of terrestrial higher plants may help plants resist drought stress through multiple physiological mechanisms. Extraradical mycelium of the AM fungal symbionts may be able to enter finer pores than root hairs and thus enhance transport and uptake of nutrients (especially $\mathrm{P}$ ) and water from fine pores (Marulanda et al. 
2003; Khalvati et al. 2005). Moreover, enhancement of the $P$ status in mycorrhizal plants is essential for plant growth and development, which is indirectly related to improvement of plant tolerance to drought stress (Davies et al. 1992). AM fungi may also directly improve plant water relations by increasing root water absorption and leaf water potential, regulating transpiration rate (Augé 2001, 2004), and enhancing photosynthetic rates (Subramanian and Charest 1995). The underlying mechanisms for the aforementioned effects of mycorrhiza on water uptake may be the changes in plant drought-related gene expression, such as genes encoding aquaporins (Ruiz-Lozano et al. 2006; Porcel et al. 2006; Li et al. 2013) or genes encoding 9-cis-epoxycarotenoid dioxygenase (NCED), the key enzyme in the biosynthesis of abscisic acid (ABA) (Aroca et al. 2008). The latter authors also reported that Rhizophagus intraradices significantly enhanced the expression of $n c e d$ and other plant genes encoding plasma membrane intrinsic proteins (PIPs) in roots and resulted in a remarkable increase of shoot dry weight under drought conditions. Furthermore, it has recently been demonstrated that two functional aquaporins from $R$. intraradices were responsible for a significant increase in the root relative water content of maize plants ( $\mathrm{Li}$ et al. 2013). Therefore, AM fungi play important roles in plant adaptation to drought stresses.

The relative importance of mycorrhizal fungi and root hairs in plant P uptake and growth differs between studies. Some studies have shown that fine and dense root systems, or long root hairs which could function similarly to AM fungal hyphae in $\mathrm{P}$ transport, are responsible for a decreased dependency of plants on mycorrhizal fungi (Baylis 1975; Baon et al. 1994; Schweiger et al. 1995). However, extraradical mycelium has a greater $\mathrm{P}$ uptake capacity than root hairs, even when they have similar lengths, because well-dispersed hyphae can enter smaller soil pores and do not compete with each other for P (Barber 1984; Baldwin et al. 1972; Jakobsen et al. 2005). In contrast, some other studies have indicated long root hairs to be more important than mycorrhiza for P uptake from soil (Baon et al. 1994). Although there have been many studies focusing on the beneficial effects of AM fungi and root hairs on plant growth, less information concerning the relative importance of AM fungi and root hairs in plant drought tolerance is available. It has been suggested that AM fungal hyphae could increase contact between soil and roots (Davies et al. 1992) and absorb water and P more effectively than root hairs in drying soils (Tisdall 1991; Augé 2001), when cavitation in drying soils blocked water movement from soil to the roots.

In spite of the likely superiority of AM fungi to root hairs in $\mathrm{P}$ and water uptake, a direct comparative study on root hair and AM fungal efficiency under drought conditions is still lacking. For the present study, a hairless root barley mutant and its wild-type parent were chosen to test the hypothesis that AM fungi could compensate for the absence of root hairs and effectively promote plant growth by improvement of plant water status and $\mathrm{P}$ uptake under drought stresses.

\section{Materials and methods}

Host plants and AM fungus

Seeds of wild-type $(w t)$ and a root-hairless mutant (bald root barley, brb) of Hordeum vulgare L. cv. Pallas (Gahoonia et al. 2001) were soaked for $3 \mathrm{~h}$ in tap water and pregerminated on moist filter paper for $72 \mathrm{~h}$. Both barley genotypes were grown with or without inoculation of the AM fungus $R$. intraradices Błaszk, Wubet, Renker, and Buscot (recently renamed from Glomus intraradices Schenck \& Smith by Schüßler and Walker 2010) isolate AH01. The inoculum was obtained from Beijing Academy of Agriculture and Forestry Sciences, China, and consisted of sand, spores, mycelia, and colonized root fragments.

\section{Growth medium}

Experimental soil was collected from Duolun County $\left(42^{\circ} 02^{\prime}\right.$ $\left.\mathrm{N}, 116^{\circ} 17^{\prime} \mathrm{E}\right)$, Inner Mongolia, China; it had a pH $(1: 2.5, \mathrm{~m} / \mathrm{v})$ of 8.17 and final extractable $\mathrm{P}$ content (extracted by $0.5 \mathrm{~mol} \mathrm{l}^{-1} \mathrm{NaHCO}_{3}$ ) of $6.73 \mathrm{mg} \mathrm{kg}^{-1}$. The growth medium for barley plants was a mixture of river sand and soil $(1: 9, w / w$, particle size $<2 \mathrm{~mm}$ ) that were sterilized by autoclaving at $121^{\circ} \mathrm{C}$ for $1 \mathrm{~h}$ on two consecutive days. Before sowing, basal nutrients of $25 \mathrm{mg} \mathrm{P} \mathrm{kg}{ }^{-1}\left(\mathrm{KH}_{2} \mathrm{PO}_{4}\right), 120 \mathrm{mg} \mathrm{K} \mathrm{kg}^{-1}$ $\left(\mathrm{KH}_{2} \mathrm{PO}_{4}\right.$ and $\left.\mathrm{K}_{2} \mathrm{SO}_{4}\right)$, and $90 \mathrm{mg} \mathrm{N} \mathrm{kg}{ }^{-1}\left(\mathrm{NH}_{4} \mathrm{NO}_{3}\right)$ were thoroughly mixed into the growth medium together with $30 \mathrm{~g}$ inoculum (ca. 1,800 spores) per pot. For the non-inoculated controls, equivalent sterilized inoculum was mixed with the growth medium together with a 5-ml filtrate of the AM fungal inoculum to control for microbial populations (except for AM fungal propagules) in different inoculation treatments.

\section{Experimental procedure}

PVC columns ( $7 \mathrm{~cm}$ in diameter and $18 \mathrm{~cm}$ in height) were filled with $900 \mathrm{~g}$ growth medium and three barley seeds sown in each. Seedlings were thinned to one per container at 6 days after emergence. For the establishment of two water regimes, half of the containers (both inoculated and non-inoculated) were subjected to drought stress in which the soil water content was maintained at $8 \%$ on dry soil basis (approx. $35 \%$ field water capacity), while the other half were maintained under well-watered conditions $(15 \%$ water content equating to $70 \%$ field water capacity) throughout the entire experiment. The water loss, adjusted based on container weight and estimated plant weight, was daily supplemented to maintain the desired moisture content by a weighing 
method. There were in total eight treatments (full combinations of mycorrhizal status, barley genotypes, and water regimes) with four replicates for each treatment. The resulting 32 containers were set up in a completely randomized block design. The experiment was conducted in a controlled environment growth chamber at $700 \mu \mathrm{mol} \mathrm{m} \mathrm{s}^{-2}$ light intensity, $16: 8 \mathrm{~h}$ and $25: 20{ }^{\circ} \mathrm{C}$ (day/night), and $70 \%$ relative humidity.

Parameters measured

\section{Leaf gas exchange and water use efficiency (WUE)}

At 37 days after seedling emergence, net $\mathrm{CO}_{2}$ assimilation rate $\left(P_{\mathrm{n}}\right)$, leaf transpiration rate $\left(T_{\mathrm{r}}\right)$, and stomatal conductance $\left(G_{\mathrm{s}}\right)$ were measured in situ on the third mature leaf with a portable photosynthesis system (Li-6400, Li-COR, USA). During the period of measurements, a 6400-02B LED source provided a photosynthetic photon flux density (PFD) of $1,300 \mu \mathrm{mol} \mathrm{m}{ }^{-2} \mathrm{~s}^{-1}$. Measurements were performed $2 \mathrm{~h}$ after soil water content of each container had been brought back to the desired level, in a random order during the late morning (09:00-11:00 a.m.) and early afternoon (1:00-3:00 p.m.), and $\mathrm{CO}_{2}$ concentration in the growth chamber was maintained at $350 \mathrm{~g} \mathrm{~m}^{-3}$. During these measurements, there was approximately $0.5-0.6 \%$ water loss (on dry soil basis) for all treatments. Leaf area was calculated as follows: the measured region of the leaf was detached and scanned using Founder AnyScan Z700 (Founder Technology Group Corp., China). The corresponding images were analyzed with Adobe Photoshop CS (Adobe Systems, San Jose, CA, USA). WUE was calculated according to the following equation: $\mathrm{WUE}={ }^{P_{\mathrm{n}}} / T_{\mathrm{r}}$.

\section{Leaf water potential ( $\Psi)$}

At 40 days after seedling emergence, $\Psi$ was determined using a SKPM 1400 pressure chamber (Skye Instruments Ltd., Powys, UK) under the same conditions as stated above. In order to ensure good tightness, the base of the third isolated mature leaf was fixed with soft tapes and squeezed into pores of rubber seals. The rubber seal was thereafter fastened, and $\Psi$ was recorded when there was continuous water efflux from the leaf base, depending on a pressure bottle to increase the chamber pressure.

\section{Biomass production}

At 42 days after seedling emergence, plant shoots and roots were separately harvested. The adhering soil particles were washed away from roots with deionized water. Shoot and root dry weights were recorded after drying samples at $70{ }^{\circ} \mathrm{C}$ for $48 \mathrm{~h}$.
Symbiosis development and hyphal length density (HLD)

Prior to drying, approximately $1 \mathrm{~g}$ of fresh roots as a subsample was cleared in $10 \% \mathrm{KOH}$ and stained with $0.05 \%$ trypan blue in lactic acid $(v / v)$ (Phillips and Hayman 1970) to confirm the presence of fungal structures (intraradical mycelia, vesicles, and arbuscules) that were identified at $\times 200$ magnification under a light microscope. The mycorrhizal colonization was calculated according to the root segment frequency method and expressed as a percentage of the length of root segments containing AM fungal structures (Biermann and Linderman 1981). Extraradical hyphae were extracted from $4 \mathrm{~g}$ growth medium, and HLD was determined by a grid-line intercept technique (Jakobsen et al. 1992). Intersections between trypan blue-stained hyphae and a grid in the eyepiece were counted in 25 random fields of view at $\times 200$ magnification under a light microscope, and HLD was calculated according to the method described by Tennant (1975). All data on hyphal length density were corrected for background levels obtained from non-inoculated treatments.

\section{P concentration analysis}

About $0.2 \mathrm{~g}$ ground plant samples were added into a digestion tube, and $10 \mathrm{ml}$ guaranteed reagent $\mathrm{HNO}_{3}$ was used to digest plant samples for $12 \mathrm{~h}$, followed by a microwave-accelerated reaction for $35 \mathrm{~min}$ in a Microwave-Accelerated Reaction System (CEM, USA). The digested solution was diluted to $50 \mathrm{ml}$. Solution P concentrations were determined using a microplate reader (SPECTRA max 190, MD, USA). Tenmicroliter solution was reacted with the molybdenum stibium anti-mix reagent for $20 \mathrm{~min}$ at $42{ }^{\circ} \mathrm{C}$ and analyzed at a wavelength of $820 \mathrm{~nm}$. P concentration was determined based on a standard $P$ curve at $820 \mathrm{~nm}$.

\section{Mycorrhizal and root hair dependency}

The dependency of $b r b$ on $R$. intraradices under the same water regime was calculated according to Klironomos (2003), based on the following equation: mycorrhizal dependency $=\left(1-\left[b n / \sum a\right]\right) \times 100 \%$, where $b$ is the mean total dry weight of $b r b$ under non-inoculated treatment, $n$ is the number of $b r b$ treatments, and $a$ is the mean total dry weight of the $b r b$ treatment. Based on a mycorrhizal dependency calculation, the following equation was deduced for root hair dependency under the same water regime: root hair dependency $=\left(1-\left[{ }^{B N} / \sum A\right]\right) \times 100 \%$, where $B$ is the mean total dry weight of $b r b$ under non-inoculated treatment, $N$ is the number of non-inoculated treatments, and $A$ is the mean total plant dry weight of noninoculated treatment. 


\section{Statistical analysis}

Data were expressed as the mean $\pm \mathrm{SD}$ of observations. Twoway ANOVA was performed for the data on mycorrhizal colonization values and hyphal length density to compare barley genotypes and soil water regimes, whereas three-way ANOVA was performed for the data of the remaining parameters to compare mycorrhizal status, barley genotypes, and soil water regimes using SPSS (version 13.0, SPSS Inc., USA). Based on ANOVA output, Duncan's multiple-range test (Duncan 1955) was performed to make comparisons between treatments. When there were no significant interactions, data were subjected to $t$ test in Microsoft ${ }^{\circledR}$ Excel 2003 to test for the significant differences between non-inoculated $w t$ and $b r b$ or between non-inoculated and inoculated $w t / b r b$ under drought stress.

\section{Results}

Colonization of roots and soil by $R$. intraradices

Mycorrhizal colonization of both $w t$ and $b r b$ increased, while HLD decreased, under drought stress. There were significant interactions between water regimes and genotypes both on mycorrhizal colonization $(P<0.01)$ and on HLD $(P<0.05)$. Under the well-watered condition, average mycorrhizal colonization of $b r b(46 \%)$ was the same as that of $w t(46 \%)$, while inoculated $b r b$ produced more extraradical hyphae $\left(1.96 \mathrm{~m} \mathrm{~g} \mathrm{~g}^{-1}\right)$ than inoculated $w t\left(1.83 \mathrm{~m} \mathrm{~g}^{-1}\right)$. In contrast, average mycorrhizal colonization of $b r b(62 \%)$ was markedly higher $(P<0.05)$ than that of wt $(49 \%)$ under drought stress, though $R$. intraradices in association with $b r b$ produced less extraradical hyphae, compared to $w t\left(1.33 \mathrm{~m} \mathrm{~g}^{-1}\right.$ versus $\left.1.63 \mathrm{~m} \mathrm{~g}^{-1}, P<0.05\right)$.

Plant growth

In general, there was no significant difference in plant dry weights of $w t$ between non-inoculated and inoculated treatments regardless of water regimes, while $R$. intraradices promoted $b r b$ growth, especially for root dry weight $(P<0.05)$ irrespective of soil water regimes (Fig. 1a, b; Table 1). Dry weights of $b r b$ were significantly increased by $R$. intraradices; non-inoculated $w t$ and inoculated $b r b$ exhibited similar dry weights under drought stress (Fig. 1a, b). In the absence of the AM fungus, the $w t$ plants showed markedly higher dry matter production than $b r b(P<0.05)$ under both water regimes.

Mycorrhizal and root hair dependency

Based on a control of non-inoculated $b r b$, a positive contribution of mycorrhiza and root hairs to plant growth was quantified. Under the well-watered condition, root hair dependency of barley (40\%) was higher than mycorrhizal dependency (18\%) (Fig. 2). However, the barley mutant showed almost the same dependency on the AM fungus (46\%) as on root hairs $(52 \%)$ under drought stress.

\section{Plant $\mathrm{P}$ nutrition}

Mycorrhizal fungal inoculation and barley genotypes showed significant influences on both shoot and $\operatorname{root} \mathrm{P}$ concentrations, and water regime had a significant influence on root $\mathrm{P}$ concentration $(P<0.05$; Table 1$)$. Apart from the root $\mathrm{P}$ concentration of $b r b$ under well-watered conditions, both $w t$ and $b r b$ benefited from inoculation with $R$. intraradices. $R$. intraradices showed more pronounced influence on $\mathrm{P}$ uptake under drought stress than under well-watered conditions, and $b r b$ in association with $R$. intraradices possessed a higher $\mathrm{P}$ concentration than inoculated $w t$ under drought conditions (Fig. 1c, d). In the absence of the AM fungus, root hairs significantly increased shoot $\mathrm{P}$ concentration but simultaneously decreased root $\mathrm{P}$ concentration irrespective of the water regime.

\section{$\Psi$ and WUE}

The AM symbiosis significantly increased $\Psi$ of $b r b$ under drought stress $(P<0.05)$ (Fig. 3; Table 1). There were no significant differences between inoculated $w t$ and $b r b$. In the absence of $R$. intraradices, root hairs did not mitigate the negative influence of drought on $\Psi$ (Fig. 3). The AM fungus increased WUE of $b r b$ (Fig. 1h) and had a significant influence on WUE of $b r b$ under drought stress $(P<0.05$; Table 1$)$. WUE was unaffected by root hairs for the non-inoculated plants (Table 1).

\section{Leaf gas exchange}

There was no significant influence of $R$. intraradices on $P_{\mathrm{n}}, T_{\mathrm{r}}$, and $G_{\mathrm{s}}$ in $w t$ regardless of water regimes, except for $P_{\mathrm{n}}$ under drought stress. The presence of mycorrhiza significantly enhanced $P_{\mathrm{n}}$ under both water regimes and enhanced $T_{\mathrm{r}}$ and $G_{\mathrm{s}}$ under drought stress for the $b r b$ mutant (Fig. 1e-g). Overall inoculation effects were highly significant $(P<0.01)$ for $P_{\mathrm{n}}$ and $T_{\mathrm{r}}$ and significant $(P<0.05)$ for $G_{\mathrm{s}}($ Table 1$)$. For nonmycorrhizal plants, only $P_{\mathrm{n}}$ was significantly higher in $w t$ with root hairs than in $b r b$ under drought stress.

\section{Discussion}

Drought causes water loss in plant tissues and adversely affects crop growth. It is estimated that $28 \%$ of the Earth's land surface has low water potential and hampers crop 
Fig. 1 Effects of mycorrhizal inoculation and water regime on shoot (a) and root (b) dry weight, shoot (c) and root (d) $\mathrm{P}$ concentration, net $\mathrm{CO}_{2}$ assimilation rate $\left(P_{\mathrm{n}}\right)(\mathbf{e})$, stomatal conductance $\left(G_{\mathrm{s}}\right)(\mathbf{f})$, transpiration rate $\left(T_{\mathrm{r}}\right)(\mathbf{g})$, and water use efficiency (WUE) (h) of wildtype barley $(w t)$ and a root hairdeficient mutant $(b r b)$. AM fungus used was $R$. intraradices (Ri). The error bars represent SD $(n=4)$. Asterisks indicate significant differences $(P<0.05)$ between non-inoculated $w t$ and $b r b$ under drought stress. Hash indicates significant differences $(P<0.05)$ between non-inoculated and inoculated $b r b$ under drought stress
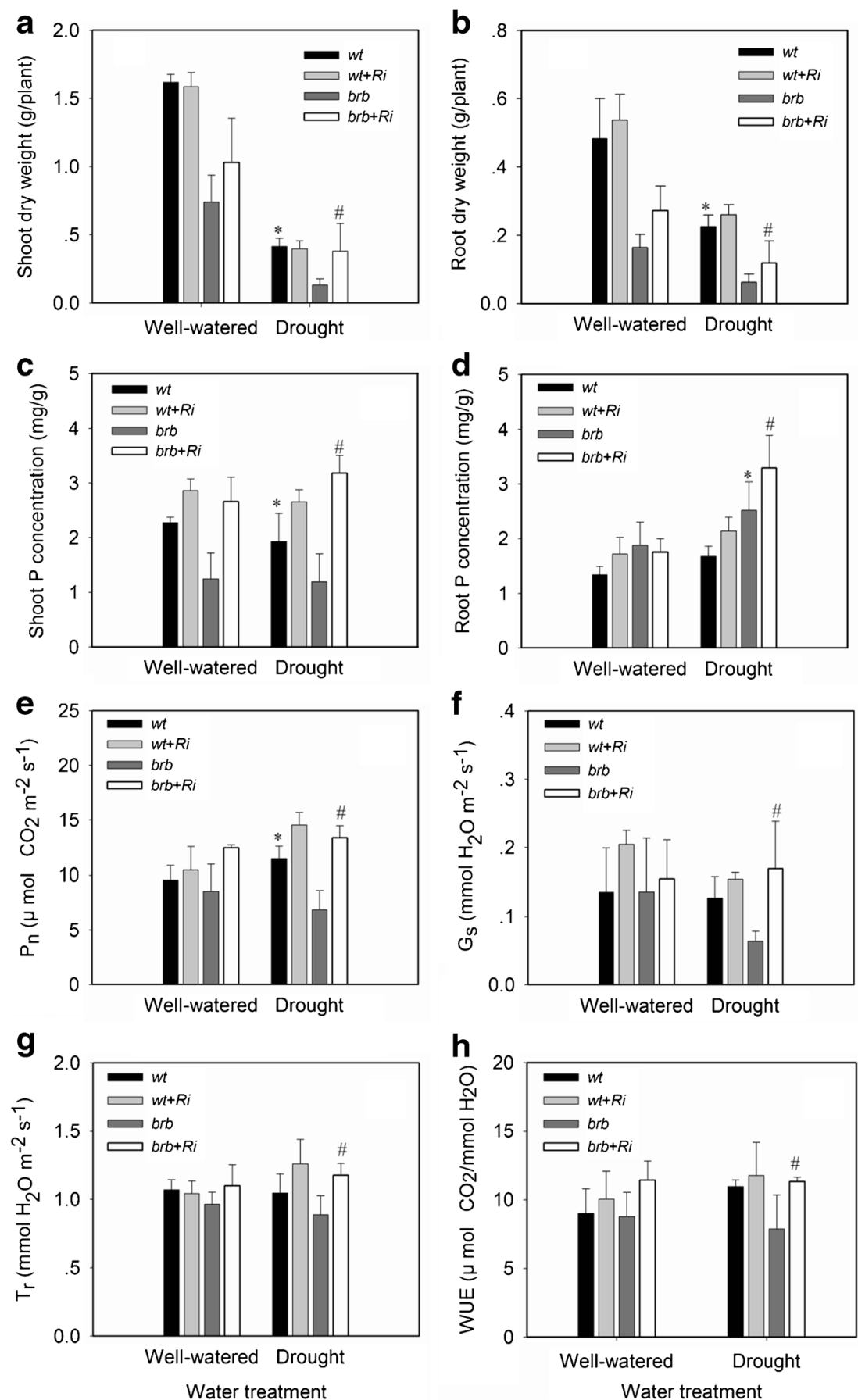

production (Jones 2007; Bray 2004). It has been demonstrated that AM fungi and root hairs take an active part in the protection of plants against drought stress (Augé 2001; Ruiz-Lozano 2003; Brown et al. 2012). However, there has been no direct comparison on the importance of AM fungi and root hairs in plant drought tolerance, as plant species used in previous studies naturally have root hairs (Schweiger et al. 1995). Recently, Brown et al. (2013) reported that root hairs were more effective in $\mathrm{P}$ acquisition than AM fungi under Pdeficient conditions by using barley mutants exhibiting different root hair lengths. Although Brown et al. (2012) also demonstrated that root hair length was important for shoot $P$ accumulation and plant growth under drought stress, there was no clarification of the relative importance of AM fungi and root hairs in plant drought tolerance. Using a monogenic hairless root mutant of barley and its wild type, the present study provides first and direct evidence to support the hypothesis that both AM fungi and root hairs are conducive to plant drought tolerance. Under the same experimental conditions, barley showed almost the same dependency on $R$. intraradices 
Table 1 Results of three-way ANOVA showing the statistical significance of the effect of mycorrhizal inoculation and water regime on dry weight, $\mathrm{P}$ concentration, leaf water potential $(\Psi)$, net $\mathrm{CO}_{2}$ assimilation rate
$\left(P_{\mathrm{n}}\right)$, stomatal conductance $\left(G_{\mathrm{s}}\right)$, transpiration rate $\left(T_{\mathrm{r}}\right)$, and water use efficiency (WUE) of wild-type barley $(w t)$ and a root hair-deficient mutant $(b r b)$

\begin{tabular}{|c|c|c|c|c|c|c|c|c|c|}
\hline & \multicolumn{2}{|c|}{ Dry weight } & \multicolumn{2}{|c|}{$\mathrm{P}$ concentration } & \multirow[t]{2}{*}{$P_{\mathrm{n}}$} & \multirow[t]{2}{*}{$G_{\mathrm{s}}$} & \multirow[t]{2}{*}{$T_{\mathrm{r}}$} & \multirow[t]{2}{*}{ WUE } & \multirow[t]{2}{*}{$\Psi$} \\
\hline & Shoot & Root & Shoot & Root & & & & & \\
\hline Inoculation (I) & ns & $* *$ & $* *$ & $*$ & $* *$ & $*$ & $* *$ & $*$ & ** \\
\hline Genotype (G) & $* *$ & $* *$ & $*$ & $* *$ & ns & ns & ns & ns & ns \\
\hline Water (W) & $* *$ & $* *$ & ns & $* *$ & ns & ns & ns & ns & ** \\
\hline $\mathrm{I} \times \mathrm{G}$ & ns & ns & $* *$ & ns & $*$ & ns & ns & ns & ns \\
\hline $\mathrm{I} \times \mathrm{W}$ & ns & ns & ns & ns & ns & ns & ns & ns & ** \\
\hline $\mathrm{G} \times \mathrm{W}$ & $* *$ & $* *$ & ns & $*$ & $*$ & ns & ns & ns & ns \\
\hline $\mathrm{I} \times \mathrm{G} \times \mathrm{W}$ & ns & ns & ns & ns & ns & ns & ns & ns & ** \\
\hline
\end{tabular}

$n s$ not significant

Significance by three-way ANOVA: $* * P<0.01 ; * P<0.05$

as on root hairs under drought stress. The results also revealed potentially different underlying mechanisms between AM fungi and root hairs in regulation of plant drought tolerance. $R$. intraradices enhanced plant drought tolerance mainly by improving uptake of water and $\mathrm{P}$, while root hairs probably through an improvement of shoot $\mathrm{P}$ nutrition status.

AM fungal colonization can increase plant $\mathrm{P}$ acquisition and improve plant growth (Davies et al. 1993). In terms of $P$ nutrition in this study, apart from root $\mathrm{P}$ concentration of $b r b$ under well-watered conditions, $R$. intraradices increased $\mathrm{P}$ concentration in both $w t$ and $b r b$ but had a greater influence on $b r b$, especially under drought conditions. The higher dependency of $b r b$ on the AM fungus indicates that it partly compensated for root hairs in $\mathrm{P}$ uptake by the mutant, though drought significantly decreased HLD in $b r b$. P uptake has previously been found to be more closely related to HLD than root colonization (Duan et al. 2011). However, the specific

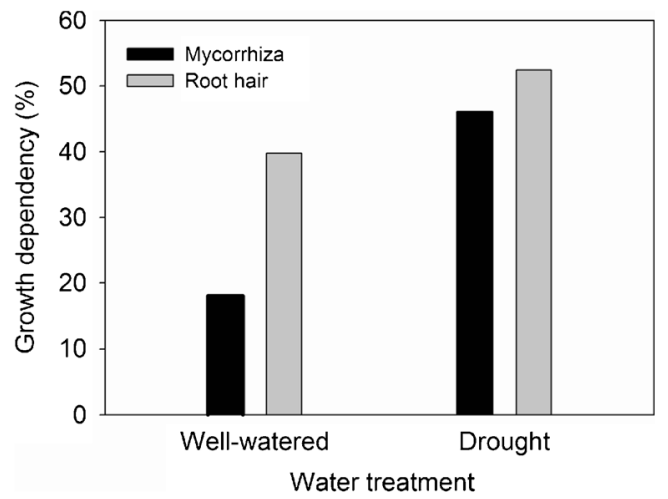

Fig. 2 Mycorrhizal and root hair dependency of barley plants under different water regimes. The mycorrhizal dependency was calculated based on biomass change of inoculated compared to non-inoculated root hair-deficient barley mutant $(b r b)$. The root hair dependency was calculated based on biomass change of non-inoculated wild type compared to non-inoculated $b r b$ ability of AM fungal hyphae to acquire $\mathrm{P}$ probably compensated for the damaged hyphal network under drought stress.

In non-inoculated treatments, root hairs increased shoot $\mathrm{P}$ concentration while decreasing root $\mathrm{P}$ concentration. Gahoonia and Nielsen (2003) showed that low-P environments are necessary for root hairs to absorb $\mathrm{P}$ whereas they might be dispensable under high $\mathrm{P}$ conditions. Root hairs could increase $\mathrm{P}$ use efficiency and enhance barley tolerance to $P$ deficiency under drought stress (Brown et al. 2012). In the present study, there might have been a relatively high $\mathrm{P}$ content for $w t$ in the growth medium. Bates and Lynch (2001) stated that at high P availability, wild-type and hairless mutant plants were equal in growth and $\mathrm{P}$ acquisition, which was also confirmed by Chen et al. (2005). Under high P availability, root hairs might even impose negative influences on plant performance (Bates and Lynch 2001). Another possibility is that competition exists between root hairs for $\mathrm{P}$, which means not all the root hairs of $w t$ plants were responsible for P uptake from soil (Barber 1984; Baldwin et al. 1972;

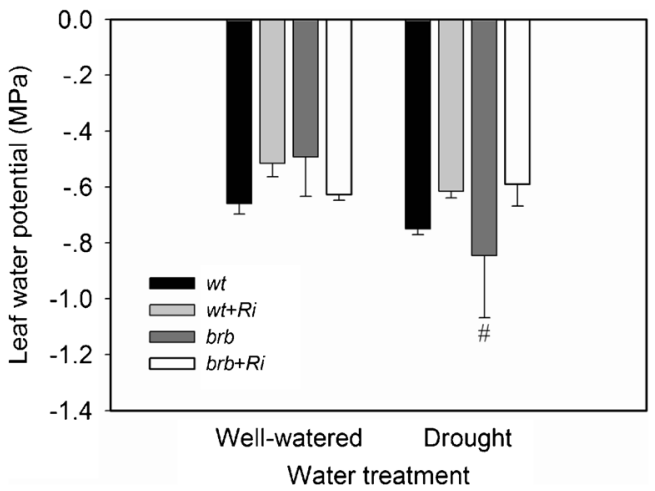

Fig. 3 Effects of mycorrhizal inoculation and water regime on leaf water potential of wild-type $(w t)$ barley and a root hair-deficient mutant $(b r b)$. AM fungus used was $R$. intraradices $(R i)$. The error bars represent SD $(n=4)$. Hash indicates significant difference $(P<0.05)$ between noninoculated and inoculated $b r b$ under drought stress 
Jakobsen et al. 2005). Despite this, wt barley plants showed a greater capacity for $\mathrm{P}$ uptake and transferred more $\mathrm{P}$ from roots to shoots than $b r b$, judging from the higher shoot to root ratio of $\mathrm{P}$ allocation, whether the plants were under wellwatered or drought conditions.

Photosynthesis is one of the primary physiological processes for plant growth and development (Sheng et al. 2008). A previous study has reported that AM fungi improved gas exchange under drought stress (Wu and Xia 2006). Likewise, the present study also showed that mycorrhizal plants had higher $P_{\mathrm{n}}, T_{\mathrm{r}}$, and $G_{\mathrm{s}}$ than the corresponding nonmycorrhizal plants. In accordance with the positive effects of AM fungi on plant growth and $\mathrm{P}$ concentration, $R$. intraradices was more important for $b r b$ in improving $P_{\mathrm{n}}, T_{\mathrm{r}}$, and $\mathrm{G}_{\mathrm{s}}$ under drought stress. Although root hairs contributed to the improvement of gas exchange, the influence of root hairs was not as significant as that of the AM fungus. Gas exchange has been reported to correlate with uptake and transport of water and nutrients (Zhu et al. 2010). It can be speculated from the present study that, based on shoot $\mathrm{P}$ concentration, $\mathrm{P}$ uptake by both root hairs and $R$. intraradices was conducive to an increase in gas exchange. However, according to the plant water status, unlike root hairs, efficient uptake of water by the AM symbiosis also contributed to an increased photosynthesis rate.

Water status largely influences plant growth and physiological processes. The AM symbiosis can improve tissue water content and leaf physiology and affect the plant water relations under drought stress (Augé 2001). The regulation of plant water status by the symbiosis may relate to host phenology, P and carbon nutrition (Augé 2001). More carbon assimilation per unit water transpired, i.e., greater photosynthetic capacity, can increase water use efficiency and enhance tolerance of mycorrhizal seedlings to osmotic stress (Nandy et al. 2007). The results from the present study also show that $R$. intraradices increased WUE of both $w t$ and $b r b$ barley plants and had more profound impacts on WUE of $b r b$ under drought conditions. At the same time, the AM fungus had a significant influence on $\Psi$, an important trait that influences plant water status, of the $b r b$ mutant under drought stress. Although root hairs remarkably increased plant growth, $P_{\mathrm{n}}$, and shoot $\mathrm{P}$ concentration under drought stress, this was not related to the improvement of plant water status, as no significant increase in $\Psi$ and WUE by root hairs was observed. Therefore, $R$. intraradices was more favorable to the maintenance of plant water balance than root hairs under drought stress. The beneficial effects of root hairs on plant performance may result from the uptake of other nutrients, considering corresponding transporters that are localized in root hairs, and deserves further study.

In conclusion, the present results suggest that both AM fungi and root hairs improve plant tolerance to drought stress. $R$. intraradices enhanced plant drought tolerance most likely by improving $\mathrm{P}$ uptake and plant water relations, as indicated by increased leaf water potential and WUE, which subsequently led to an increased photosynthetic capacity. Root hairs, on the other hand, promoted plant growth and photosynthetic capacity under the drought condition probably by improving uptake of nutrients, including P. As suggested by Gleeson (1993), root/shoot ratios could be used to describe the carbon source and sink balance in plants. Under drought stress, the inoculated $b r b$ mutant had a lower root/shoot ratio (0.31) than the non-inoculated $w t$ plants $(0.54)$, which implies less carbon costs for sustaining plant growth with the AM fungal partner than with root hairs. Further research is necessary to compare the physiological functions of root hairs and AM fungi especially under environmental stress conditions.

Acknowledgments We are very grateful to Dr. Iver Jakobsen for providing barley seeds and to Dr. Erik Verbruggen for his critical reading of the manuscript. The study was financially supported by the National Natural Science Foundation of China (41371264), Knowledge Innovation Program of the Chinese Academy of Sciences (Project no. KZCX2YW-BR-17), and the National Key Technology R\&D Program (2012BAC25B03).

\section{References}

Aroca R, Alguacil MM, Vernieri P, Ruiz-Lozano JM (2008) Plant responses to drought stress and exogenous ABA application are modulated differently by mycorrhization in tomato and an ABAdeficient mutant (Sitiens). Microb Ecol 56:704-719

Augé RM (2001) Water relations, drought and vesicular-arbuscular mycorrhizal symbiosis. Mycorrhiza 11:3-42

Augé RM (2004) Arbuscular mycorrhizae and soil/plant water relations. Can J Soil Sci 84:373-381

Baldwin JP, Tinker PB, Nye PH (1972) Uptake of solutes by multiple root systems from soil. 2. Theoretical effects of rooting density and pattern on uptake of nutrients from soil. Plant Soil 36:693-708

Baon JB, Smith SE, Alston AM (1994) Growth responses and phosphorus uptake of rye with long and short root hairs: interactions with mycorrhizal infection. Plant Soil 167:247-254

Barber SA (1984) Soil nutrient bioavailability—a mechanistic approach. Wiley-Interscience, New York

Bates TR, Lynch JP (2001) Root hairs confer a competitive advantage under low phosphorus availability. Plant Soil 236:243-250

Baylis GTS (1975) The magnolioidmycorrhiza and mycotrophy in root systems derived from it. In: Sanders FE, Mosse B, Tinker PB (eds) Endomycorrhizas. Academic Press, New York, pp 373-389

Biermann B, Linderman RG (1981) Quantifying vesicular-arbuscular mycorrhizae: a proposed method towards standardization. New Phytol 87:63-67

Bray EA (1997) Plant responses to water deficit. Trends Plant Sci 2:4854

Bray EA (2004) Genes commonly regulated by water-deficit stress in Arabidopsis thaliana. J Exp Bot 55:2331-2341

Brechenmacher L, Lee J, Sachdev S, Song Z, Nguyen THN, Joshi T, Oehrle N, Libault M, Mooney B, Xu D, Cooper B, Stacey G (2009) Establishment of a protein reference map for soybean root hair cells. Plant Physiol 149:670-682

Brown LK, George TS, Barrett GE, Hubbard SF, White PJ (2013) Interactions between root hair length and arbuscular mycorrhizal 
colonisation in phosphorus deficient barley (Hordeum vulgare). Plant Soil 372:195-205

Brown LK, George TS, Thompson JA, Wright G, Lyon J, Dupuy L, Hubbard SF, White PJ (2012) What are the implications of variation in root hair length on tolerance to phosphorus deficiency in combination with water stress in barley (Hordeum vulgare)? Ann Bot 110: 319-328

Chen BD, Roos P, Borggaard OK, Zhu YG, Jakobsen I (2005) Mycorrhiza and root hairs in barley enhance acquisition of phosphorus and uranium from phosphate rock but mycorrhiza decreases root to shoot uranium transfer. New Phytol 165:591-598

Davies FT, Potter JR, Linderman RG (1993) Drought resistance of mycorrhizal pepper plants independent of leaf P-concentration response in gas exchange and water relations. Physiol Plant 87:45-53

Davies FT, Potter JR, Linderman RG (1992) Mycorrhiza and repeated drought exposure affect drought resistance and extraradical hyphae development of pepper plants independent of plant size and nutrient content. J Plant Physiol 139:289-294

Denby K, Gehring C (2005) Engineering drought and salinity tolerance in plants: lessons from genome-wide expression profiling in Arabidopsis. Trends Biotechnol 23:547-552

Duan TY, Facelli E, Smith SE, Smith FA, Nan ZB (2011) Differential effects of soil disturbance and plant residue retention on function of arbuscular mycorrhizal (AM) symbiosis are not reflected in colonization of roots or hyphal development in soil. Soil Biol Biochem 43: $571-578$

Duncan DB (1955) Multiple range and multiple F-tests. Biometrics 11:142

Gahoonia TS, Nielsen NE (2003) Phosphorus (P) uptake and growth of a root hairless barley mutant (bald root barley, brb) and wild type in low- and high-P soils. Plant Cell Environ 26:1759-1766

Gahoonia TS, Nielsen NE, Joshi PA, Jahoor A (2001) A root hairless barley mutant for elucidating genetic of root hairs and phosphorus uptake. Plant Soil 235:211-219

Gleeson SK (1993) Optimization of tissue nitrogen and root-shoot allocation. Ann Bot 71:23-31

Jakobsen I, Abbott LK, Robson AD (1992) External hyphae of vesiculararbuscular mycorrhizal fungi associated with Trifolium subterraneum. 1. Spread of hyphae and phosphorus inflow into roots. New Phytol 120:371-380

Jakobsen I, Chen BD, Munkvold L, Lundsgaard T, Zhu YG (2005) Contrasting phosphate acquisition of mycorrhizal fungi with that of root hairs using the root hairless barley mutant. Plant Cell Environ 28:928-938

Jones HG (2007) Monitoring plant and soil water status: established and novel methods revisited and their relevance to studies of drought tolerance. J Exp Bot 58:119-130

Jupp AP, Newman EI (1987) Morphological and anatomical effects of severe drought on the roots of Lolium Perenne L. New Phytol 105: 393-402

Khalvati MA, Hu Y, Mozafar A, Schmidhalter U (2005) Quantification of water uptake by arbuscular mycorrhizal hyphae and its significance for leaf growth, water relations, and gas exchange of barley subjected to drought stress. Plant Biol 7:706-712

Klironomos JN (2003) Variation in plant response to native and exotic arbuscular mycorrhizal fungi. Ecology 84:2292-2301

Lan WZ, Wang W, Wang SM, Li LG, Buchanan BB, Lin HX, Gao JP, Luan S (2010) A rice high-affinity potassium transporter (HKT) conceals a calcium-permeable cation channel. Proc Natl Acad Sci U S A 107:7089-7094

Li T, Hu YJ, Hao ZP, Li H, Wang YS, Chen BD (2013) First cloning and characterization of two functional aquaporin genes from an arbuscular mycorrhizal fungus Glomus intraradices. New Phytol 197:617-630

Libault M, Brechenmacher L, Cheng JL, Xu D, Stacey G (2010) Root hair systems biology. Trends Plant Sci 15:641-650
Loqué D, Yuan L, Kojima S, Gojon A, Wirth J, Gazzarrini S, Ishiyama K, Takahashi H, von Wirén N (2006) Additive contribution of AMT1;1 and AMT1;3 to high-affinity ammonium uptake across the plasma membrane of nitrogen-deficient Arabidopsis roots. Plant J 48:522534

Marulanda A, Azcón R, Ruiz-Lozano JM (2003) Contribution of six arbuscular mycorrhizal fungal isolates to water uptake by Lactuca sativa plants under drought stress. Physiol Plant 119:526-533

Maruyama-Nakashita A, Nakamura Y, Yamaya T, Takahashi H (2004) Regulation of high-affinity sulphate transporters in plants: towards systematic analysis of sulphur signalling and regulation. J Exp Bot 55:1843-1849

Nandy P, Das S, Ghose M, Spooner-Hart R (2007) Effects of salinity on photosynthesis, leaf anatomy, ion accumulation and photosynthetic nitrogen use efficiency in five Indian mangroves. Wetl Ecol Manag $15: 347-357$

Oldroyd GE, Downie JA (2006) Nuclear calcium changes at the core of symbiosis signaling. Curr Opin Plant Biol 9:351-357

Phillips JM, Hayman DS (1970) Improved procedure of clearing roots and staining parasitic and vesicular-arbuscular mycorrhizal fungi for rapid assessment of infection. Trans Br Mycol Soc 55:159-161

Porcel R, Aroca R, Azcón R, Ruiz-Lozano JM (2006) PIP aquaporin gene expression in arbuscular mycorrhizal Glycine max and Lactuca sativa plants in relation to drought stress tolerance. Plant Mol Biol 60:389-404

Ruiz-Lozano JM (2003) Arbuscular mycorrhizal symbiosis and alleviation of osmotic stress. New perspectives for molecular studies. Mycorrhiza 13:309-317

Ruiz-Lozano JM, Porcel R, Aroca R (2006) Does the enhanced tolerance of arbuscular mycorrhizal plants to water deficit involve modulation of drought-induced plant genes? New Phytol 171:693-698

Schünmann PH, Richardson AE, Smith FW, Delhaize E (2004) Characterization of promoter expression patterns derived from the Pht1 phosphate transporter genes of barley (Hordeum vulgare L.). J Exp Bot 55:855-865

Schüßler A, Walker C (2010) The glomeromycota: a species list with new families and new genera 1-58. Libraries at The Royal Botanic Garden Edinburgh, The Royal Botanic Garden Kew, Botanische Staatssammlung Munich, and Oregon State University. Available at: www.amf-phylogeny.com

Schweiger PF, Robson AD, Barrow NJ (1995) Root hair length determines beneficial effect of a Glomus species on shoot growth of some pasture species. New Phytol 131:247-254

Segal E, Kushnir T, Mualem Y, Shani U (2008) Water uptake and hydraulics of the root hair rhizosphere. Vadose Zone $\mathrm{J}$ 7: $1027-1034$

Sheng M, Tang M, Chen H, Yang BW, Zhang FF, Huang YH (2008) Influence of arbuscular mycorrhizae on photosynthesis and water status of maize plants under salt stress. Mycorrhiza $18: 287-296$

Subramanian KS, Charest C (1995) Influence of arbuscular mycorrhizae on the metabolism of maize under drought stress. Mycorrhiza 5: 273-278

Tennant D (1975) A test of a modified line intersect method of estimating root length. J Ecol 63:995-1001

Tisdall JM (1991) Fungal hyphae and structural stability of soil. Aust J Soil Res 29:729-743

White RG, Kirkegaard JA (2010) The distribution and abundance of wheat roots in a dense, structured subsoil-implications for water uptake. Plant Cell Environ 33:133-148

Wu QS, Xia RX (2006) Arbuscular mycorrhizal fungi influence growth, osmotic adjustment and photosynthesis of citrus under well-watered and water stress conditions. J Plant Physiol 163:417-425

Zhu CX, Song BF, Xu HW (2010) Arbuscular mycorrhizae improves low temperature stress in maize via alterations in host water status and photosynthesis. Plant Soil 331:129-137 